\title{
Cortical Blindness Following Neonatal Hypoxic Ischaemic Encephalopathy: Cases Series from Bamako
}

\author{
Elien Gagnan Yan Zaou Tou Rodrigue Romuald*, Bakayoko Seydou, Sidibe Mohamed Kolé, \\ Konikpo Ali, Diallo Hammadoun, Toure Ousmane, Sidibe Tata
}

Institute of African Tropical Ophthalmology, University of Sciences and Technology of Bamako, Bamako, Mali

Email: *rodrigueelien@yahoo.fr

How to cite this paper: Romuald, E.G.Y.Z.T.R., Seydou, B., Kolé, S.M., Ali, K., Hammadoun, D., Ousmane, T. and Tata, S. (2021) Cortical Blindness Following Neonatal Hypoxic Ischaemic Encephalopathy: Cases Series from Bamako. Open Journal of Ophthalmology, 11, 99-104. https://doi.org/10.4236/ojoph.2021.112008

Received: January 29, 2021

Accepted: May 11, 2021

Published: May 14, 2021

Copyright $\odot 2021$ by author(s) and Scientific Research Publishing Inc. This work is licensed under the Creative Commons Attribution International License (CC BY 4.0).

http://creativecommons.org/licenses/by/4.0/

\begin{abstract}
Purpose: Report of series of cases of cortical blindness that occurred after neonatal hypoxic-ischaemic by analysing its epidemiological frequency in black Africa. Methodology: This is the report of two clinical cases received in consultation on Monday $16^{\text {th }}$ November 2020 and Thursday $7^{\text {th }}$ January 2021 in the paediatric ophthalmology department of the IOTA-University Hospital. Results: They are two infants, aged 05 and 17 months respectively, who were brought in for consultation by their mother for lack of eye-tracking movement since birth. Both infants were born at term following a dystocic delivery. At birth, both infants had a very poor Apar score and were given a resuscitation treatment. The clinical examination coupled with the results of the paraclinical examinations allowed us to conclude at cortical blindness induced the neonatal hypoxic-ischaemic encephalopathy. Their therapeutic management, in collaboration with the neurologist, included the combination of piracetam suspension and Valproate sodium syrup. The evolution after three months of treatment is marked by the regression of epileptic seizures and the perception of light. Conclusion: In black Africa, neonatal hypoxic-ischaemic encephalopathy is the second leading cause of cortical blindness in children, after the neuromalaria sequels.
\end{abstract}

\section{Keywords}

Cortical Blindness, Neonatal Hypoxic Ischaemic Encepalopathy, Bamako

\section{Introduction}

Neonatal hypoxic-ischemic encephalopathy (NHIE) is a serious brain injury resulting from a significant decrease in blood flow and oxygen to the brain at birth. 
Thus, hypoxic-ischemic encephalopathy is oxygen deprivation that generally occurs close to labour and delivery. Neonatal hypoxic-ischemic encephalopathy (NHIE) remains an important cause of mortality in preterm children and, in term children, is responsible for acute neurological injury leading to long-term neurodevelopmental disability thereafter [1]. The risk of disability and impaired cognitive development correlates with the severity of NHIE [1] [2]. The incidence of NHIE varies from 1.5 per 1000 live births in Europa to 14.9 per 1000 live births in sub-Saharan Africa. We report two cases of cortical blindness induced by the NHIE and we will discuss the epidemioclinical and evolutionary profiles of the cortical blindness due to the NHIE in black Africa.

\section{Observation}

\subsection{Case 1}

She is a 17-month-old female infant, the youngest of four siblings from nonconsanguineous marriages. In her history we note the notion of pregnant high blood pressure in her mother treated with and her delivery was laborious with an Apgar side score of 3 at the first minute. She benefited from neonatal resuscitation (external cardiac massage, ventilation, warming, vitamin K1 injection and local care) for 15 minutes. Her anthropometric parameters at birth were: weight $=3150 \mathrm{~g}$, height $=48 \mathrm{~cm}$, head circumference $=35 \mathrm{~cm}$. The history of the disease reveals that the infant would have started the convulsive attacks from the third day of life, treated with the injectable medicines of which the parents were unaware. The lack of visual tracking movements, observed by the parents from birth until the $17^{\text {th }}$ month of life, was the reason for consulting our department for treatment. At the clinical examination on Monday 16 November 2020, the infant had a lack of interest in light stimulation, the corneas are transparent, the pupils are symmetrical and reactive, the fundus is normal. The rest of the somatic examination objectified a delay in psychomotor development characterised by poor head posture (Figure 1(a)). We called in the neuro paediatrician. Infectious tests (haemogram, CRP, the study of the cerebrospinal fluid) were negative, the transfontanellar ultrasound showed a generalised cerebral and cortical atrophy (Figure 1(b)), the electroencephalogram trace was in favour of the epileptic encephalopathy of the infant (Figure 1(c)). The confirmation of the anamnestic, clinical and paraclinical arguments allowed us to conclude cortical blindness induced by NHIE. It was treated with piracetam syrup: $100 \mathrm{mg}$ every 12 hours and sodium valproate syrup: $50 \mathrm{mg}$ every 12 hours. He was prescribed paediatric physiotherapy sessions combined with monthly clinical monitoring. There was no clinically detectable improvement in the first two months of follow-up. By the third month, the infant was able to become interested in visual stimulation. Oculomotricity was maintained in all quadrants of the visual field. Clinical examination of both eyes was normal. The orbitocerebral CT scan requested, concluded that there was no expansive orbitocerebral process and no detectable anoxic sequellae lesions (Figure 1(d)). We instituted semi-annual (once) and 

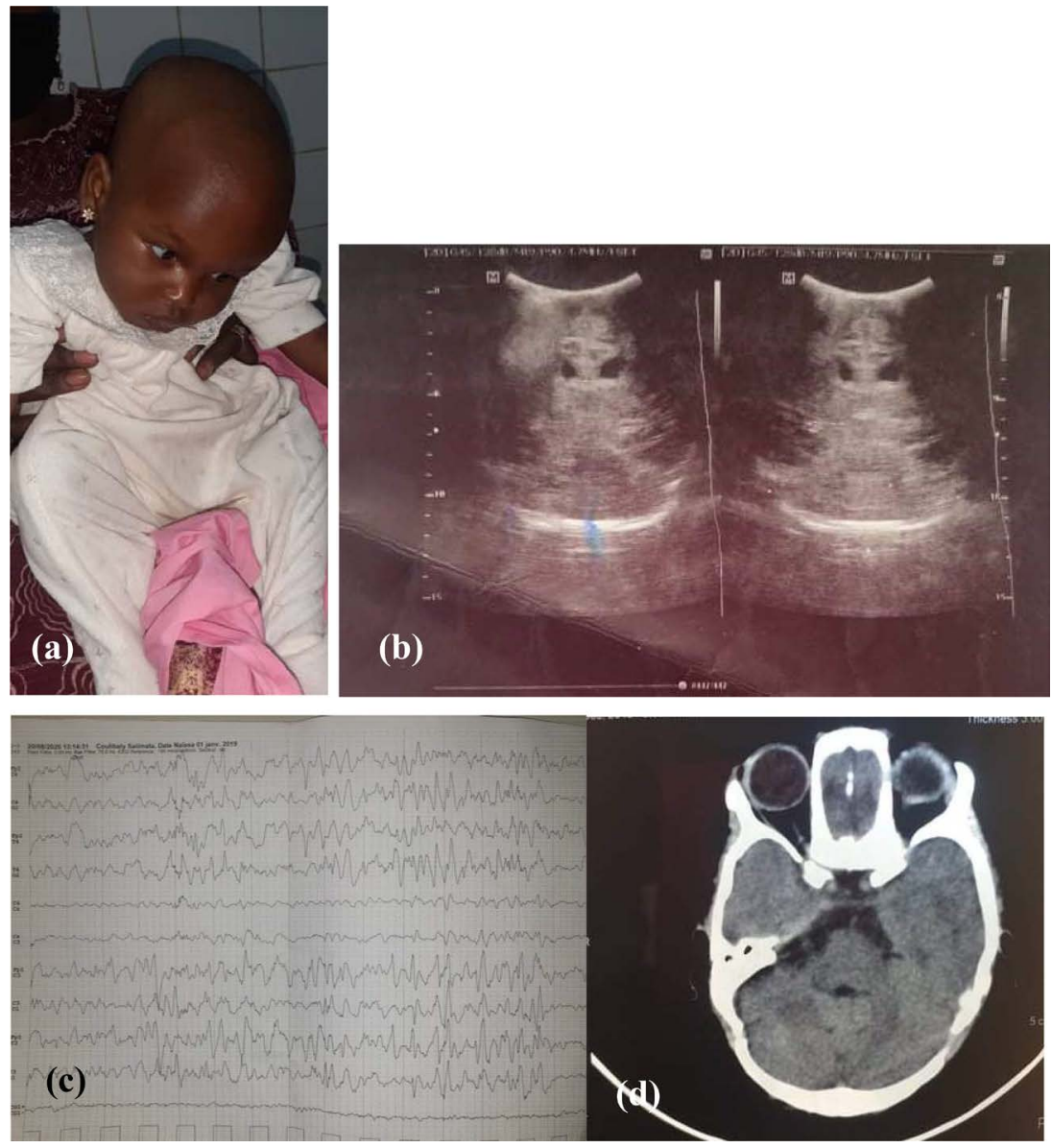

Figure 1. (a) Infant with axial hypotonia; (b) transfontanel ultrasound showing generalised cerebral and cortical atrophy; (c) tracing of the electroencephalogram in favour of the infant's epileptic encephalopathy; (d) orbital brain CT scan free of anoxo-ischaemic sequellae.

then annual follow-up.

\subsection{Case 2}

Male infant, aged 05 months, the only child of the family, from non-consanguineous marriage. He has a history of acute foetal distress. Childbirth was dystocic. His Apgar score at the first minute of life was rated at $5 / 10$. He was resuscitated for 15 minutes. His anthropometric parameters at birth were: weight $=3500 \mathrm{~g}$, height $=43 \mathrm{~cm}$, head circumference $=32 \mathrm{~cm}$. The infant was brought by his parents to consult us for visual wandering. The clinical examination on Thursday $7^{\text {th }}$ January 2021 found the absence of eye-tracking movements of the visual stimulation, the corneas were clear, the pupils were symmetrical and reactive, the fundus was normal in both eyes. There was also an associated delay in psychomotor acquisition (Figure 2(a)). The orbitocerebral tomodensitometry carried out on January $9^{\text {th }}, 2021$ found an important cortico-cerebral atrophy sus tentorial with a focus of anoxo-ischemic anoxo-ischemic sequellae (Figure 2(b) \& Figure 2(c)). In front of this picture, and with the cooperation of the neuro 

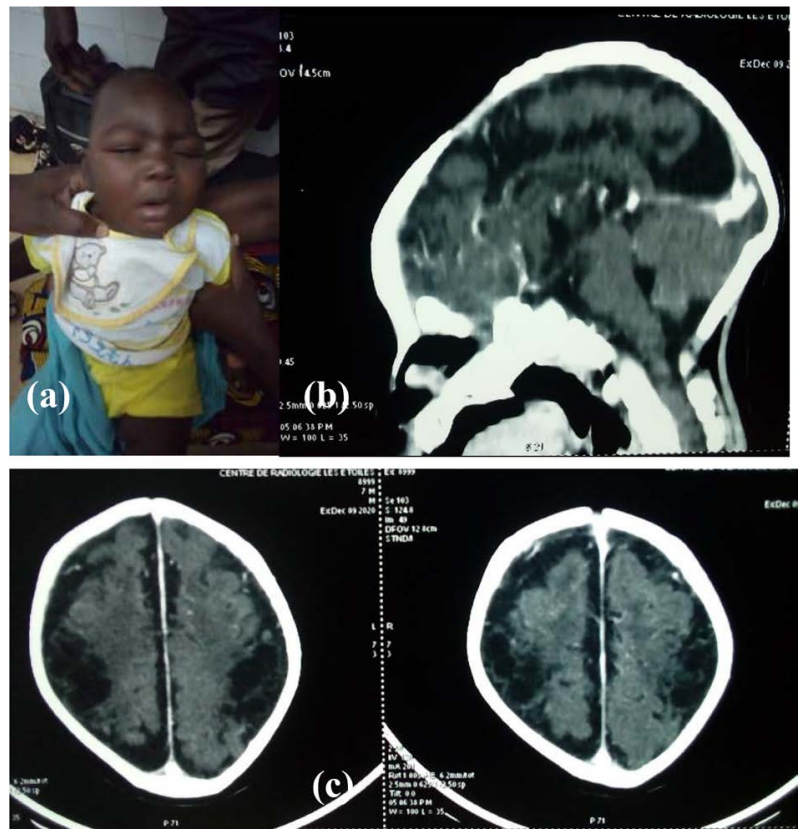

Figure 2. (a) the infant with axial hypotonia; ((b), (c)) the sagittal and coronal sections of the orbitocerebral tomography showing cortical-subcortical hypodensity in favour of cortical-subcortical atrophy.

paediatrician, we have retained the diagnosis of cortical blindness secondary to NEHI. We established the following therapeutic protocol piracetam syrup: 100 mg every 12 hours and sodium valproate syrup: $50 \mathrm{mg}$ every 12 hours. He was prescribed paediatric physiotherapy sessions combined with monthly clinical monitoring. We plan to see the child again in three months time (i.e. April $6^{\text {th }}$ 2021).

\section{Discussion}

In developed countries, the primary cause of visual disorders in children is central neuro visual impairment [3]. These central neuro visual disorders are the result of cerebral anoxia (or even better, hypoxia) [3]. Cerebral anoxia can be observed either in cases of prematurity or in children born at term [3].

The epidemiology of NHIE in Europe is better-known thanks to the early diagnosis and therapeutic management [3].

In Africa, the published data are disparate [2] [4].

In Sub Saharan Africa, neuromalaria has been frequently reported as the primary cause of cortical blindness in children [4]. On the other hand, for South African authors, the NHIE is reported to be the primary cause of cortical blindness in children in sub-Saharan Africa (2).

The low ratio of cases of child cortical blindness in sub-Saharan Africa does not allow us to formally define its epidemiology in Black Africa.

It is, therefore, necessary for national eye health programmes to consider the management of child cortical blindness as a priority.

Better knowledge of the epidemiological profile of child cortical blindness in 
Black Africa would facilitate the implementation of strategies for early diagnosis and improved survival rates.

The differential diagnosis of cortical blindness is made with psychogenic blindness and previous blindness [3].

Cortical blindness is differentiated from psychogenic blindness by the abolition of optokinetic nystagmus [3].

In anterior blindness, the abolition of pupillary reflexes differentiates it from cortical blindness [3].

In our two patients, the presence and vivacity of pupillary reflexes rule out the hypothesis of anterior blindness by bilateral damage to the pre-geniculated visual pathways.

Nowadays, there is no standardised and systematic management strategy for cortical blindness [3].

Rehabilitation plays a key role in the management of hemianoptic patients [3].

Black Africa would gain enormously in the early detection and management of all situations likely to lead to neonatal cerebral anoxia (prematurity, maternal-fetal infections, in utero growth retardation, dystocic deliveries, etc.).

In both reported cases, nonchalance in the management of situations at risk of perinatal cerebral anoxia was the cause of cortical blindness.

\section{Conclusion}

The epidemiology of NHIE in black Africa is not known. Of the few published works, the sequelae of neuromalaria would be the most frequent cause of cortical blindness in black African children. Early detection and improved neonatal resuscitation would give better results.

\section{Ethical Consideration}

The study was approved by the research committee of the Institute of African Tropical Ophthalmology, University of Sciences and Technology of Bamako.

Parental consent has been obtained for the publication of their children's images.

\section{Conflicts of Interest}

The authors declare no conflicts of interest regarding the publication of this paper.

\section{References}

[1] Shalak, L. and Perlman, J.M. (2004) Hypoxic-Ischemic Brain Injury in the Term Infant-Current Concepts. Early Human Development, 80, 125-141.

https://doi.org/10.1016/j.earlhumdev.2004.06.003

[2] Ballot, D.E., Rakotsoane, D., Cooper, P.A., Ramdin, T.D., Chirwa, T. and Pepper, M.S. (2020) A Prospective Observational Study of Developmental Outcomes in Survivors of Neonatal Hypoxic Ischaemic Encephalopathy in South Africa. South African Medical Journal, 110, 308-312. https://doi.org/10.7196/SAMJ.2020.v110i4.14311 
[3] Touré, A., Diakité, A.A., Sylla, M., Ombotimbe. A., Maiga, B., et al. (2015) Sequelles du neuropaludisme dans le service de pediatrie du CHU Gabriel Toure (Bamako, Mali). Mali Medical, 30, 14-18.

[4] Chokron, S. (2013) Cécité corticale. EMC Ophtalmologie, 23, 1-8. https://doi.org/10.1016/S0246-0343(06)41709-3 